\title{
TURISMO Y TIC. NOTAS SOBRE LOS PROCESOS RECIENTES DE TECNIFICACIÓN DE LOS DESTINOS TURÍSTICOS
}

Francisco López Palomeque*

Universidad de Barcelona

\section{RESUMEN}

En el texto se aportan unas notas sobre los procesos recientes de tecnificación de los destinos turísticos, bajo el marco de la relación entre el turismo y las tecnologías de la información y la comunicación (TIC). En concreto, se analiza el proceso de creación y difusión de los conceptos y de los discursos en torno a los paradigmas ciudad inteligente y destino inteligente. Se analiza el papel que tienen los diversos agentes en dicho proceso (la academia, los agentes del mercado y las instituciones) y la percepción de los distintos actores urbanos sobre la relación turismo-TIC, constatándose la existencia de una visión crítica a los discursos dominantes, visión fundamentada en la óptica social, más allá de la óptica tecnológica. Para ilustrar el alcance de la tecnificación de las ciudades turísticas y el debate en torno al tema, se aportan referencias a la situación en Barcelona, tanto a los hechos que suceden en la ciudad y en su gobierno como a las reflexiones que diversos analistas han realizado a partir lo que sucede en la ciudad condal y en otras ciudades.

Palabras clave: Ciudad, turismo, TIC, ciudad inteligente, destino inteligente, Barcelona

\section{ABSTRACT}

Tourism and ICT. Notes on the recent technification process of tourist destinations.

The text provides some notes on the recent processes of modernization of tourist destinations, within the framework of the relationship between tourism an information and communications technology (ICT). Specially, the process of creation and dissemination of concepts and discourses about paradigms smart city and smart destination is analyzed. The role played by different actors in this process (the academy, market players

*1opez.palomeque@ub.edu 
and institutions) and the perception of the different urban actors on the tourism-ICT relationship are analyzed, confirming the existence of a critical view of the dominant discourses, a view based on the social optics, beyond the technological perspective. To illustrate the scope of the modernization of the tourist towns and debate on the issue, references are given to the situation in Barcelona, both events occurring in the city and its government and reflections that various analysts have made from what is happening in Barcelona and other cities.

Keywords: City, Tourism, ICT, smart city, smart destination, Barcelona

\section{PRESENTACión}

La relación entre el turismo y la tecnología se fundamenta en la propia naturaleza del turismo. La experiencia turística, como experiencia vital, comprende un conjunto de actividades en la esfera de la producción - consumo, cuya evolución ha estado vinculada con los avances de todo tipo de tecnologías y el proceso contemporáneo de tecnificación de la sociedad. Turismo es, además, desplazamiento entre origen y destino; y en esta dimensión se enfatiza la importancia que la tecnología ha tenido en el desarrollo geo-histórico del turismo (extensión territorial, evolución del fenómeno), destacando los avances en medios e infraestructuras de transporte. Turismo es, además, información y comunicación. Y en esta dimensión las Tecnologías de la Información y Comunicación (TIC) han provocado una verdadera revolución del turismo, en el marco del paradigma de la Sociedad de la Información, vigente en el siglo XXI.

Las TIC, y también otros tipos de tecnologías, no solo afectan al turismo, sino que obviamente la relación de causa-efecto ha de enmarcarse en la interrelación del conjunto de actividades socioeconómicas - de las que el turismo es una más- con las tecnologías, sus innovaciones y sus avances. Interesa considerar este hecho por cuanto el turismo es un fenómeno transversal en su dimensión económica. Por otra parte, en todos los componentes del sistema turístico se proyectan los avances tecnológicos, y en particular de las TIC. Y lo mismo ocurre con todos los subsistemas y componentes del sistema funcional urbano.

La incorporación del concepto sistema turístico y sus componentes conduce a considerar el concepto destino turístico, y su asociación con la realidad urbana, las ciudades y el sistema funcional urbano, que constituye una categoría analítica fundamental para abordar el rol de las TIC en los destinos. Por su carácter instrumental, las TIC afectan a todos y cada uno de los componentes del sistema funcional urbano y del sistema turístico. Esta afectación a todos los componentes y a sus complejas interrelaciones supone, por un lado, una 
dificultad analítica sobre el alcance de la relación entre TIC y destino turístico; $\mathrm{y}$, por otro, lado, una dificultad en identificar los ámbitos de actuación y contenidos de las políticas relacionadas con el tema y el alcance de gestión pública sobre el conjunto del destino turístico o sobre algunos de sus elementos.

Son diversas las cuestiones que confluyen en las relaciones entre tecnología y turismo y diversos los resultados, ya sean en el sistema y estructura física (morfología) como en el sistema y estructura funcional. Un aspecto destacado lo constituye la diferencia entre las ciudades, en función de la entrada de tecnología. En este sentido cabe afirmar que el desarrollo de las redes tecnológicas ha constituido tanto un factor de modernización del espacio urbano como un factor de determinación de la jerarquía urbana y, recientemente con la irrupción de las TIC, uno de los factores clave de la función de liderazgo en un escenario de competencia entre ciudades.

En este marco general, el presente trabajo aporta unas notas sobre los procesos recientes de tecnificación de los destinos turísticos, en particular sobe los denominados destinos turísticos inteligentes. El tema es de gran actualidad, desde diversas perspectivas y ámbitos (sectorial, político-institucional, académico, empresarial, etc.). Y una de las motivaciones últimas para abordar esta cuestión ha sido la aparición en 2015 de dos publicaciones sobre el tema, aportaciones destacadas por sus enfoques, en cada caso, que inducen a su vez a la reflexión y debate.

Por una parte, la obra de Blanco (2015), titulada Libro Blanco de los destinos turísticos inteligentes. Estrategias y soluciones para fomentar la innovación en el turismo digital; y, por otra parte, la publicación Destinos turísticos inteligentes. Manual Operativo para la Configuración de Destinos Turísticos Inteligentes, realizada por el Instituto Universitario de Investigaciones Turísticas (IUTT), de la Universidad de Alicante, por encargo del Instituto Valenciano de Tecnologías Turísticas (INVAT-TUR), de la Generalitat Valenciana.

Para abordar el objetivo señalado, el texto se ha estructurado en cuatro apartados. En el primero de ellos se hace alusión a los marcos de referencia (evolución del turismo, paradigmas sociales y nueva conceptualización), haciendo énfasis a la aparición de nuevos conceptos. El segundo apartado se dedica al proceso de formación y difusión de dichos conceptos. Posteriormente, en el tercer apartado se abordan las cuestiones relacionadas con el sistema urbano, sistema turístico y el papel de las TIC; y, finalmente, se indaga sobre la realidad compleja, los actores y el gobierno del destino turístico. Las «notas» finalizan con unas breves consideraciones que sintetizan las ideas desarrolladas a lo largo del texto. 
MARCOS DE REFERENCIA: EVOLUCIÓN DEL TURISMO, PARADIGMAS SOCIALES Y NUEVA CONCEPTUALIZACIÓN

\section{Evolución del turismo: cambio del turismo y nuevos paradigmas}

En la producción científica sobre la historia del turismo se observan diferentes propuestas de periodización y de identificación de fases, que caracterizan la evolución del fenómeno turístico (Vera -coord-, 2013). Al margen de los rasgos específicos de cada una de las fases lo que cabe subrayar es el carácter cambiante y dinámico del turismo. Su última fase -la actual- se caracteriza por una segmentación de los mercados, la flexibilidad en los factores de producción, distribución y consumo, la necesaria explotación de sinergias y economías de profundización en la producción y activación en el mercado para alcanzar unos niveles de rentabilidad aceptables. Y, obviamente, el impacto de las tecnologías de la información y comunicación ha sido un factor de cambio y caracterización.

Las transformaciones que se han operado en la demanda turística y en la oferta turística -en el contexto de la sociedad postindustrial- han propiciado, a finales del siglo xx y a principios del XxI, la aparición de un nuevo escenario turístico, que se conceptualiza como nuevo paradigma turístico, al tiempo que han cuestionado la hegemonía del modelo convencional y los espacios turísticos tradicionales. El nuevo turismo es un reflejo del nuevo paradigma social sobre el que no hay unanimidad en su designación. Se habla de sociedad postfordista, sociedad del ocio, sociedad del espectáculo, sociedad de la información, sociedad del conocimiento, sociedad tecnológica o sociedad inteligente (smart society), entre otros.

La nueva conceptualización se observa tanto en la esfera de la definición de los paradigmas sociales como, a nivel más concreto, en la descripción y explicación de la nueva realidad turística compleja, en sus nuevas manifestaciones específicas, en sus diversos elementos que en muchas ocasiones requieren una nueva terminología y el uso de nuevas acepciones.

\section{Nueva conceptualización}

En esta ocasión, el interés se centra en la conceptualización asociada a la relación turismo-nuevas tecnologías de la información y comunicación, y particularmente a los resultados del proceso de tecnificación de los destinos turísticos. La revisión bibliográfica permite identificar diversos términos nuevos (relativamente nuevos) y seleccionar los más próximos al objetivo de estas páginas. Buena parte de estos nuevos vocablos no aparecen en la Enciclopedia del Turismo (Jafari, 2002), lo que puede entenderse como una evidencia de su reciente irrupción. Esta selección se justifica por sus significados específicos, por el rol de su uso en la creación del discurso sobre la relación destino turísticoTIC, por su difusión y por su influencia en el imaginario social. A continuación 
se recogen de manera breve las referencias básicas a los nuevos vocablos, como paso previo para abordar posteriormente las causas de la formación de los conceptos y discursos, y quien los crea y difunde. Estos nuevos conceptos son: inteligencia turística, tecnologías de la información y la comunicación (TIC), smart city (ciudad inteligente) y smart destination (Destino Turístico Inteligente -DTI).

Inteligencia turística. Previo a la definición de inteligencia turística es la consideración del significado de inteligencia. Los rasgos conceptuales de la inteligencia giran en torno a las ideas siguientes: «capacidad de aprender, adaptarse y elaborar sentido común; de comprender la época y sociedad en las que vivimos e interactuar con sus entornos, y de combinar conocimientos para lograr objetivos en una amplia variedad de entornos» (Blanco, 2015). Lo inteligente (lo smart), a pesar de sus difusos perfiles, pudiera conceptuarse como "adaptación de la idea original de inteligencia a nuestro tiempo y a sus capacidades tecnológicas». Sin duda, es una cualidad o un principio a modo de símbolo contemporáneo que inunda buena parte de nuestras vidas. Una opción para saber el significado del concepto inteligencia turística es recurrir a aquellos ámbitos en los que se utiliza, por ejemplo portales en internet o instituciones que ofrecen programas formativos. Por ejemplo, se puede consultar http://inteligenciaturistica.com/ que-es-inteligencia-turistica/; o también http://escuela.inteligenciaturistica. $\mathrm{com} /$. Para definir inteligencia turística se puede considerar la acepción genérica de inteligencia y, además, añadir que es la combinación de aplicar metodologías y tecnologías unidas al expertise y validación de los datos.

Tecnologías de la información y la comunicación (TIC). Los diccionarios y enciclopedias definen las tecnologías de la información y la comunicación (unión de los computadores y las comunicaciones) como un conjunto de servicios, redes, software y aparatos que tienen como fin la mejora de la calidad de vida de las personas dentro de un entorno, y que se integran a un sistema de información interconectado y complementario. Sin duda, las TIC han provocado una explosión en las formas de comunicarse desde principios de los años 90. Y desde entonces Internet ha pasado de ser un instrumento específico de la comunidad científica a ser una red de fácil uso que ha modificado las pautas de interacción social.

Smart city (ciudad inteligente). Las smart cities surgen de los estudios realizados para la aplicación de las Tecnologías de la Información y las Comunicaciones con el fin de resolver las necesidades de la ciudad actual; y se definen como: «aquellas ciudades que unan las TIC para hacer que, tanto su infraestructura crítica, como sus componentes y servicios públicos ofrecidos, sean más interactivos, eficientes y los ciudadanos puedan ser más conscientes de ellos» (Fundación Telefónica, 2011). Se subraya que la aplicación de las políticas que lleven a la conversión de una ciudad en una smart city no se basa 
únicamente en la voluntad de la administración, sino que requiere de la implicación de todos los agentes que conviven en la ciudad.

Smart destination (destino turístico inteligente -DTI). Los smart destinations o DTI son resultado de la aplicación de los principios de las smart cities al destino turístico, un concepto vinculado a la innovación en la gestión de las ciudades, la eficiencia en la gestión de los servicios y la mejora en la calidad de vida en las ciudades con la implantación de las TIC y el seguimiento de principios como la sostenibilidad y la accesibilidad en la gestión (Marrero y Santana, 2008). Actualmente, existe una falta de concreción a la hora de definir las características de los DTI, por lo que todavía hoy parece necesaria una propuesta de definición, que debe tener presente dos diferencias esenciales respecto a las smart cities: el ámbito de actuación y los beneficiarios.

En el Libro Blanco de los destinos turísticos inteligentes, Blanco (2015) realiza una amplia caracterización del DTI (algunas de sus aportaciones se abordan en el apartado siguiente) y la publicación Destinos turísticos inteligentes. Manual Operativo para la Configuración de Destinos Turísticos Inteligentes, cuando trata la definición de DTI remite a la acepción propuesta por la Sociedad Estatal para la Gestión de la Innovación y las Tecnologías Turísticas (SEGITTUR). Desde la perspectiva de SEGITTUR los Destinos Turísticos Inteligentes (DTI) se conciben como un destino turístico innovador, consolidado sobre una infraestructura de vanguardia, que garantiza el desarrollo sostenible del territorio turístico, accesible para todos, que facilita la interacción e integración del visitante con el entorno e incrementa la calidad de su experiencia en el destino. Y, en su caracterización, se añade que como consecuencia de lo anterior se estimula el incremento de la competitividad a través de la capacidad innovadora, que repercute en una mejora de la percepción del destino y en una mayor capacidad productiva de la empresa, generando una mayor calidad de vida para los residentes.

\section{LA FORMACIÓN Y DIFUSIÓN DE LOS NUEVOS CONCEPTOS}

En los últimos años los conceptos señalados en el punto anterior han irrumpido con fuerza en el mundo del turismo, afectando y revalorizando la gestión pública del turismo e induciendo el proceso de formalización del concepto destino turístico inteligente. ¿Quién crea el conocimiento sobre los nuevos hechos? ¿Quién crea los conceptos? ¿Quién los difunde y cómo se configuran los discursos sobre la relación tecnologías de la información y comunicación y turismo?; $\mathrm{y}$, concretamente sobre los destinos turísticos inteligentes. La indagación sobre estas cuestiones y las respuestas a las preguntas se han orientado a lo sucedido en el entorno próximo.

En este contexto, se han producido numerosas iniciativas institucionales que han ido definiendo un marco para el desarrollo de la relación turismo-TIC, tanto 
teórico como aplicado, que han contribuido a generalizar el paradigma de la inteligencia turística y a difundir los conceptos smart city, smart destination y otros conceptos en el nuevo turismo contemporáneo. Actualmente el concepto smart city (y conceptos e instrumentos asociados), vinculado al desarrollo de las TIC, se ha convertido en un vector indisociable de los modelos urbanos actuales y de los escenarios futuros, sea cual sea el tipo de ciudad, si bien no está exento de críticas.

En el proceso de formación, uso y difusión de estos conceptos y del discurso vinculado al correspondiente modelo de ciudad, son varios los factores y los actores que se identifican, y que actúan cada vez más interrelacionada. A continuación se identifican los tipos de actores (de forma simplificada: academia, mercado e instituciones) y posteriormente se incorporan algunas valoraciones críticas

\section{La academia}

La comunidad científica ha participado, obviamente, en la formación de los nuevos conceptos y en la formación del discurso sobre smart city y smart destination. Se observa una respuesta de la universidad, de las comunidades de científicos sociales (diversas disciplinas) sobre estos temas, a través de la creación de centros y grupos específicos de investigación, de los proyectos y publicaciones y mediante la organización de congresos y reuniones científicas. En estos últimos mecanismos también se observa una interrelación de las iniciativas con las instituciones y empresarios.

Como ejemplo y muestra de lo señalado, se puede citar la preocupación de la Asociación Española de Expertos Científicos en Turismo (AECIT) por la relación TIC-Turismo, tal como lo demuestran las ponencias de sus congresos anuales, desde mediados de la década pasada. Se pueden señalar, en particular, el XII Congreso celebrado en 2007 en Tarragona, sobre «Conocimiento, creatividad y tecnología para un turismo sostenible y competitivo»; y el XIII Congreso celebrado en Vitoria en 2008, dedicado a «Cambios tecnológicos en turismo. Producción, comercialización, innovación y territorio».

Asimismo, cabe referirse a los congresos de la Asociación de Geógrafos Españoles (AGE) y, en particular, a la labor del Grupo 10 (Grupo de Trabajo de Geografía del Turismo, Ocio y Recreación). Su último coloquio, en 2014, se dedicó a los Espacios turísticos e inteligencia territorial: respuestas ante la crisis, y reunió diversas comunicaciones en torno a las nuevas tecnologías, y en concreto sobre los destinos turísticos inteligentes en el marco de la inteligencia territorial (Luque, Caro y Zayas, 2014) y los destinos turísticos inteligentes en España como proyecto institucional o futuro del sector (Mínguez y Ruíz Jiménez, 2014). 
Por último, la tercera referencia es a las iniciativas del Instituto Universitario de Investigaciones Turísticas (IUIT) de la Universidad de Alicante, en concreto a la última: \#ICAT2014 Seminario Internacional «Innovación y Competitividad en Áreas Turísticas (ICAT2014)», dedicado a los procesos de innovación desarrollados en áreas y destinos turísticos consolidados, así como las principales líneas de investigación vinculadas a esta temática.

\section{El mercado}

Los agentes del mercado turístico -en sentido amplio- y sus dinámicas comerciales dan significados no siempre coincidentes a las palabras smart city y smart destination. Esta diferencia se explica, en parte, por el perfil de los agentes o empresas de referencia. Si las empresas son las del sector de la fabricación y prestación de servicios de las TIC, los discursos son unos; mientras que si son del sector turístico la visión es otra. En el proceso de creación del concepto destacan la iniciativas de las multinacionales de sector de la informática y de las TIC, cuya aportación es entendida por diversos analistas como creación del «producto» smart city. En este sentido se identifica la iniciativa de IBM de lanzar en 2011 el producto Intelligent Center for Smarter Cities.

Por otra parte, si se consideran a las consultoras como empresas bajo el epígrafe «mercado» (por su estrecha relación con las organizaciones, tanto empresas como instituciones) se puede afirmar que son generadoras de discursos, conceptos y metodologías sobre la visión teórica y operativa del tema; de forma similar a lo que ha ocurrido con el marco teórico, instrumentos metodológicos y praxis de planificación turística.

Los foros de debate que reúnen a los distintos tipos de actores (academia, empresarios e instituciones) han sido un reflejo de la preocupación por el impacto de las TIC en el turismo e, indirectamente, han sido mecanismos de sanción de la nueva conceptualización y medios de amplificación de la difusión de los discursos sobre el nuevo turismo y los destinos turísticos inteligentes. Quizá el ejemplo más claro sea el Foro Internacional de Turismo de Benidorm, que en varias ediciones ha abordado temas relacionados con los nuevos paradigmas, la innovación y la tecnología. El tema elegido para la edición XVII del Foro, «Estrategias para la Configuración de Destinos Turísticos Inteligentes» se centró en el modelo de destino inteligente desde la realidad actual y bajo el prisma de la oportunidad, prestando especial atención a las estrategias que permiten un desarrollo eficaz del modelo y la obtención de beneficios a corto y medio plazo.

La última muestra en este epígrafe, dedicado al rol de los agentes económicos, es la referencia al Smart City Word Expo Congress Barcelona, que se celebra en la ciudad condal desde 2011 y cuya última edición tuvo lugar los días 
18 y 19 de noviembre de 2015, reuniendo a un gran número de empresas, expertos y ciudades presentes e interesadas. El análisis del programa de 2015 ilustra de los temas de interés, y si se compara con el programa de 2011 se observan nuevas preocupaciones y problemáticas. Pero, además, interesa en esta ocasión identificar a los organismos y empresas organizadoras y colaboradoras, cuya relación constituye una verdadera fotografía de los principales agentes económicos que participan en el proceso de formación y difusión de los conceptos y discursos que fundamentan el paradigma smart city.

\section{Las instituciones}

Los actores institucionales -en sentido amplio, engloba a las distintas administraciones y organizaciones vinculadas en sus distintos niveles - juegan un papel destacado en el proceso de formación y difusión del discurso sobre la smart city y el smart destination, mediante la formulación de las políticas e instrumentos sobre el territorio (a distintas escalas), sobre el turismo y sobre las nuevas tecnologías de la información y la comunicación. Ha de señalarse, no obstante, que la intencionalidad y el enfoque del uso de los discursos y conceptos pueden moldear en cada caso sus significados.

En la última década se ha observado un progresivo incremento de iniciativas institucionales que han incorporado, de una manera u otra, el concepto smart city, y que han acabado perfilando y determinando la concepción actual de este término. Se puede hablar, pues, de un proceso de institucionalización de los conceptos smart city y smart destination y, en ese sentido, se afirma que el Destino Turístico Inteligente (DTI) en España es un proyecto institucional (Mínguez y Ruíz Jiménez, 2014).

Desde la administración central española se ha acuñado el término Destino Turístico Inteligente (DTI) para definir a los destinos donde se usan las tecnologías de vanguardia con el fin de favorecer una gestión eficiente de los recursos y la interacción del turista con el entorno todo para mejorar la calidad de la experiencia turística y en la competitividad del destino. A partir de 2010 la administración pública española -a nivel estatal- empezó a trabajar en la definición de un marco homogéneo que permitiese tecnificar los destinos turísticos bajo el concepto de Destinos Inteligentes, tal y como se planteaba inicialmente en el Plan de Turismo Español Horizonte 2020 y posteriormente en el Plan Nacional Integral de Turismo 2012-2015. Para este objetivo en el Ministerio de Industria, Energía y Turismo se encargó esta labor a dos entidades de gestión: la Sociedad Estatal para la Gestión de la Innovación y las Tecnologías Turísticas (SEGITTUR) y el Subcomité de Destinos Turísticos.

El análisis de los resultados de los últimos cinco años revela el papel clave de SEGITTUR, cuyos esfuerzos de orientan al establecimiento de una metodología 
que contemple las bases y requisitos mínimos que deberá cumplir un destino turístico para calificarlo como Destino Turístico Inteligente. Actualmente SEGITTUR es la responsable de impulsar la innovación $(\mathrm{I}+\mathrm{D}+\mathrm{i})$ tanto en el sector turístico español público (nuevos modelos y canales de promoción, gestión y creación de destinos inteligentes, etc.) como en el sector privado (apoyo a emprendedores, nuevos modelos de gestión sostenible y más competitivo, exportación de tecnología española). Este proceso cuenta siempre con la colaboración público-privada puesto que es una premisa indispensable. Además, SEGITTUR se encuentra estrechamente vinculada a TURESPAÑA donde realiza actividades centradas en el desarrollo tecnológico aplicado a la promoción y gestión del turismo. Por este perfil tecnológico, y estando las tecnologías de la información y comunicación interrelacionadas con la inteligencia, con la generación de conocimiento y, en definitiva, con las smarts cities, se determinó que esta sociedad estatal fuera la encargada de llevar a cabo el proyecto de los Destinos Turísticos Inteligentes (DTI) en España. Una descripción del proceso y su situación actual puede consultarse en el artículo de López de Ávila y García Sánchez (2015), presidente y miembro de SEGITTUR respectivamente, en la revista Economía Industrial.

El proyecto se concibió inicialmente para destinos maduros pero finalmente se eligieron destinos que se encuentran en distintos niveles de desarrollo, y con diferentes escalas, como son: Playa de Palma, El Hierro, La Gomera, Las Palmas de Gran Canaria, Haro, Castelldefels, Villajoyosa, La Axarquía, Badajoz-Elvas (España-Portugal), Jaca y Santiago de Compostela. Hasta el momento la mayoría de los proyectos piloto se encuentran en las fases de análisis y diagnóstico, realizadas en todos los casos por los técnicos de SEGITTUR y tan solo ha llegado a desarrollarse íntegramente el proyecto de la Isla del Hierro (http://www. segittur.es/es/inicio/index.html).

\section{Sistema URBANO, SISTEMA TURÍSTICO Y PAPEL DE LAS TIC}

El impacto de las nuevas tecnologías de la información y la comunicación sobre las actividades turísticas ha tenido como primera referencia interpretativa la relación TIC-turismo (como concepto holístico) y la relación TIC-ciudad, dado el peso actual del destino urbano y del concepto smart city. Sin embargo, el conocimiento más profundo y preciso obliga a incorporar dos nuevos conceptos, como categorías analíticas y relacionarlos con las TIC. Se trata de los conceptos sistema turístico (substituto en este caso del concepto turismo), y sistema funcional urbano, en vez del concepto ciudad. Este recurso conceptual y analítico operativo permite identificar de manera más rigurosa las implicaciones de las TIC en cada uno de los componentes del sistema turístico y del sistema funcional urbano. 
La interpretación sistémica del fenómeno turístico ha inducido el concepto sistema turístico, y sobre su composición se han formulado distintas propuestas. Por ejemplo, Vera -coord- (2013) identifican cuatro componentes del sistema: los turistas, los mecanismos públicos y privados, los sistemas de transportes y el destino turístico o territorio. Por su parte, Sancho -dir- (1998) contempla también cuatro componentes del sistema: la demanda, la oferta, el espacio geográfico (territorio) y, finalmente, los operadores del mercado. Sin duda, esta radiografía del sistema turístico y la naturaleza de sus compontes permite intuir que las TIC influyen (cada una de las tecnologías y sus instrumentos) de manera e intensidad distintas para cado caso. Y los ejemplos de ello son fáciles de encontrar: TIC y comportamiento de la demanda, TIC y organizaciones turísticas, TIC e intermediación y distribución y, por último, TIC y gestión de destinos turísticos, entre otros ámbitos.

A partir de este planeamiento se puede afirmar, además, que la relación TIC y los distintos componentes del sistema turístico se manifiestan de maneras muy específicas en cada caso y en ocasiones de forma singularizada (ver, por ejemplo, el estudio reciente de Parra y Santana, 2014). A este nivel, se puede hablar de situaciones sin relación, fragmentadas, frente a la percepción que pueda tenerse de una relación TIC- turismo basada y materializada en la unicidad de estos conceptos. Y esta observación es pertinente, además, por cuanto también se utiliza en un determinado nivel el concepto gestión del turismo o gestión del destino turístico inteligente, y de acuerdo con las afirmaciones anteriores estos casos aconsejan una cierta cautela ante el grado de abstracción o vaguedad de los conceptos.

En un primer nivel análisis, en el sistema urbano se identifican la forma, la función y los actores. Interesa en esta ocasión su dimensión funcional, que conduce a considerar el sistema funcional urbano, caracterizado por su complejidad, por estar formado por varios componentes interrelacionados entre si y por su diversa naturaleza.

Para profundizar en las características de los componentes del sistema funcional urbano y su relación con la tecnología y, en particular, con las TIC se puede recurrir a la obra Ciudades y ciudadanos en 2033. La transformación urbana de España, publicada en 2014 por PwC, tanto en soporte papel como en versión digital (www.pwc.es). Uno de los planteamientos de la obra es la comprensión de la ciudad como sistema funcional y su premisa es que la ciudad contemporánea puede entenderse como un complejo ecosistema de elementos o partes conectadas, en el que las actividades humanas están enlazadas por comunicaciones que interactúan en tanto el sistema evoluciona dinámicamente. En este sistema, cualquier variación o alteración en una de sus partes -ya sea espacial o estructural- origina una reacción en cadena que modifica o influye en las otras. No se olvida, por otra parte que los sistemas urbanos son abiertos, es decir, 
operan en un determinado contexto socioeconómico y natural, interactuando con él de manera constante. Ello explica, pues, que el fuerte dinamismo de los procesos urbanos junto a la intensa interacción de los cambios que tienen lugar sobre el territorio es lo que caracteriza la complejidad de las ciudades.

Con el propósito de facilitar un análisis con finalidad prospectiva de la complejidad urbana en el sistema funcional urbano se identifican diferentes subsistemas que coexisten fuertemente interrelacionados y sometidos a factores de cambio del entorno. En el estudio prospectico Ciudades y ciudadanos en 2033 el sistema funcional urbano comprende los siguientes subsistemas:

- Demanda urbana. Compuesta por ciudadanos, agentes económicos, agentes sociales y visitantes.

- Subsistema económico. Integra los agentes económicos, como los empresarios y los trabajadores; las tecnologías productivas para fabricar bienes y proporcionar servicios; los servicios productivos que prestan apoyo a las empresas y el capital para financiar sus operaciones.

- Subsistema social. Comprende aquellos elementos de la oferta urbana que tratan de satisfacer las necesidades básicas y avanzadas, tanto de la población residente como de los visitantes que acuden a la ciudad.

- Subsistema ambiental. Incluye los elementos de la oferta urbana dedicados a la preservación y el desarrollo de un medio ambiente urbano y natural acorde con las aspiraciones de la ciudadanía y de los agentes socioeconómicos.

- Subsistema de gobierno. Representa a aquellos actores y elementos de la oferta urbana que proporcionan los modelos y los instrumentos de gobernanza necesarios para lograr una armoniosa convivencia en el ámbito urbano.

- Subsistema espacial. La última dimensión de la oferta urbana es el subsistema físico-espacial. El subsistema espacial dispone de los siguientes elementos para alcanzar sus fines: sistemas de transporte; sistemas de movilidad; espacios productivos; infraestructuras básicas; áreas residenciales, equipamientos colectivos; espacios verdes y, finalmente, áreas recreativas.

Además, el sistema funcional urbano comprende el soporte tecnológico, que constituye un elemento de carácter transversal que proporciona apoyo a la operativa de los subsistemas urbanos descritos anteriormente. En otras palabras: la tecnología se ha convertido hoy en día en el soporte por excelencia para que las denominadas ciudades inteligentes puedan realizar con eficacia sus diversos procesos operativos y atender las necesidades de las demandas urbanas Por último, el carácter abierto del sistema induce a considerar los diversos factores del entorno, es decir múltiples factores geopolíticos, sociales, económicos, 
tecnológicos y políticos exógenos a la ciudad, pero que afectan a su funcionamiento y equilibrio. De hecho, los sistemas urbanos están continuamente reaccionando ante los cambios externos, tratando de adaptarse o sobreponerse a ellos. Por lo tanto, es muy importante reconocer y evaluar los factores de cambio que tienen lugar en el contexto en el que operan las ciudades (EOI, 2014).

En el caso del sistema funcional urbano, cuya composición y complejidad se ha descrito, también son aplicables las valoraciones realizadas anteriormente sobre las relaciones entre las tecnologías (particularmente las TIC) y los componentes del sistema turístico. Ahora se trataría, en cambio, de la relación entre las tecnologías y los componentes del subsistema funcional urbano señalados.

Para finalizar, cabe añadir que el desarrollo tecnológico de las ciudades, como función que determina su jerarquía en la red de ciudades, ha desplazado en determinadas escalas al rol principal que en este sentido tenía su dimensión poblacional. Este hecho confiere al desarrollo tecnológico (en particular las TIC) el rol de factor estratégico, dado que el mismo se asocia con la mejor prestación de servicios y notoriedad cultural, además de su impacto en la efíciencia de su sistema de producción-consumo.

\section{EL DESTINO: REALIDAD COMPLEJA, ACTORES Y GOBIERNO}

La relación entre tecnologías de la información y comunicación y el turismo se proyectan, finalmente, sobre el destino que, para simplificar y en consonancia con el objetivo de esta aportación, se identifica con el destino urbano, con la ciudad turística o con la ciudad con turismo.

Los discursos en torno a los conceptos smart city y smart destination son adoptados por los distintos actores urbanos y por el gobierno de numerosas ciudades como modelo a seguir o como principio para definir la gestión pública de las ciudades. Es un discurso nuevo, que se extiende y seduce. Con él se ha entrado de lleno en el marketing de destinos turísticos y, como se ha señalado en la presentación, se afirma que el desarrollo de las redes tecnológicas ha constituido tanto un factor de modernización del espacio urbano, como un factor de determinación de la jerarquía urbana y, recientemente con la irrupción de las TIC, uno de los factores clave de la función de liderazgo en un escenario o de competencia entre ciudades. No obstante, el grado de adopción y el orden de prioridad de este paradigma en el modelo de ciudad aparecen sesgados por el componente ideológico que corresponde a cada equipo de gobierno en cada caso y en cada momento. En el contexto de la gestión urbana y la gestión del destino turístico la tecnología es un medio no un fin, y por ello la componente político-ideológico, la intencionalidad de las decisiones es el factor explicativo.

La intención de este apartado, una vez presentado en las páginas anteriores el proceso de formación y difusión de los conceptos y del discurso, es recoger 
la visión crítica a los discursos dominantes, visión fundamentada en la óptica social, más allá de la óptica tecnológica. Visión crítica, además, que forma parte del debate actual sobre el tema y que últimamente se ha reactivado como consecuencia del cambio en 2015 de numerosos gobiernos municipales de España. Para ello, y siguiendo con la mirada al entorno próximo, a continuación se hace referencia a la situación en Barcelona, tanto a los hechos que suceden en la ciudad y en su gobierno como a las reflexiones que diversos analistas han realizado a partir lo que sucede en la ciudad condal y en otras ciudades.

Los días 18 al 19 de noviembre se celebró en Barcelona e Smart City Word Expo Congress, en su quinta edición desde 2011, volviendo a ser el epicentro del debate en torno al desarrollo urbano y la aplicación de tecnologías digitales en la gestión municipal. En el acto de inauguración del evento participó el Sr. Gerardo Pisarello, Primer Teniente de Alcalde de Barcelona, y una semana después publicó en la prensa de la ciudad un artículo sobre el tema, que en parte se reproduce a continuación. Su contenido va más allá de ser un hecho coyuntural o local, e ilustra las consideraciones realizadas en las líneas anteriores.

En su artículo, titulado «Ciudad inteligente, ¿para qué?» (Ara, 25 noviembre en 2015), Pisarello escribe que:

En el caso de Barcelona, demasiado a menudo, la smart city se ha pensado y diseñado de acuerdo con los intereses exclusivos de grandes corporaciones tecnológicas que operan a miles de kilómetros de la ciudad, al margen de sus necesidades específicas y de sus habitantes. Como gobierno de la ciudad, entendemos que esta lógica no puede continuar. Que tenemos la obligación de ampliar el foco del debate y de ir más allá de una noción de smart city que, como ha denunciado la socióloga Saskia Sassen, «mata la ciudad».

Si queremos hablar seriamente de smart city no podemos aceptar de manera complaciente que haya barrios donde cerca del $70 \%$ de la población no tiene acceso a internet. Y no podemos consentir, tampoco, que la ingente cantidad de datos y de información que genera la ciudad de Barcelona vaya a parar a unos pocos bolsillos privados, en vez de funcionar en código abierto, al servicio del conjunto del vecindario de la ciudad.

...los cambios tecnológicos que se han producido en los últimos años tienen un potencial indudable si lo que se quiere es construir una ciudad justa y democrática, con una economía cada vez más circular y ecológicamente sostenible. Del mismo modo, la economía digital ofrece grandes oportunidades para avanzar hacia una economía más diversificada, más cooperativa y menos dependiente de monocultivos actuales como el turismo.

\section{Y Pisarello finaliza:}

Nada de lo cual, naturalmente, se podrá hacer sin la participación activa de la ciudadanía en la coproducción de las políticas públicas y con unas instituciones demasiado permeables a intereses privados incompatibles con el bien común. Por ello es esencial rescatar el concepto de smart city de su uso meramente 
publicitario o puramente mercantil. Y la única manera de conseguirlo es vinculándolo a una ciudad de código abierto, que potencie la transparencia de la administración, que ayude a neutralizar las malas prácticas y la corrupción y que haga de la participación ciudadana, digital y presencial el núcleo de un verdadero cambio de época. No podemos concebir que una ciudad sea inteligente si no es a la vez, y por encima de todo, justa y democrática.

Barcelona abrazó el nuevo paradigma sobre la ciudad inteligente, y su dinámica y protagonismo convirtió a la ciudad a su vez en paradigma de los discursos sobre la ciudad inteligente. La visión crítica a este modelo se formaliza e institucionaliza con el cambio político en el Ayuntamiento en 2015 y con el modelo de ciudad que defiende el nuevo gobierno, tal como se deduce de los mensajes de su Primer Teniente de Alcalde. Obviamente, el posicionamiento sobre esta cuestión no surge de repente en estas fechas, sino que forma parte explicita e implícita del programa de gobierno del nuevo equipo y se ha manifestado públicamente en diversas ocasiones desde su acceso al gobierno de la ciudad. En este sentido, otra evidencia de este nuevo discurso sobre la smart city y smart destination (y sobre la relación entre tecnología y turismo, y TIC y destino turístico) son la declaraciones de otro miembro del equipo de Gobierno, Agustí Colom, concejal de Ocupación, Empresa y Turismo.

En el mismo contexto de la celebración de Smart City Word Expo Congress, Agustí Colom en declaraciones a la prensa (Jané, 2015) señalaba que el consistorio estaba reorientando la estrategia tecnológica del equipo de Trias, anterior alcalde, para resolver cuestiones prácticas de la ciudad. La smart city y los grandes proyectos vinculados a la gestión tecnológica en Barcelona ya no son una prioridad ni una bandera para la ciudad. Ahora se priorizan otras cuestiones, como la emergencia social de los desahucios y la falta de trabajo, en definitiva gestionar la ciudad desde una óptica social. Y, en este sentido, se les da una perspectiva más a corto o largo plazo a los proyectos, como los ateneos de fabricación digital (fablabs) o la gestión ciudadana a partir del big data, que eran el mantra del equipo de Urbanismo del anterior equipo de gobierno.

El debate de estas cuestiones en los medios de comunicación es rico y de él se pueden seleccionar otras aportaciones que fortalecen la visión crítica en torno al enfoque hegemónico de las TIC entendidas como un icono de exportación, una función de liderazgo exterior, sin mucho contenido sustancial y sin especificar qué lógicas urbanas hay detrás de esta apuesta tecnológica. Reproducimos en esta ocasión algunas opiniones significativas seleccionadas.

En el mes de septiembre de 2015 Jordi Borja publicó un artículo de opinión con un título explícito en relación con las interpretaciones del discurso y efectos del paradigma de la ciudad inteligente: «Smart cities, negocio o ciudadanía» (El País, 15 septiembre de 2015). En realidad se trataba de un resumen para los medios de un texto más amplio publicado en su blog. Borja, urbanista y 
geógrafo que se prodiga como colaborador de la prensa escrita, aborda habitualmente el tema de la ciudad, sus cambios, su gestión y sus conflictos. En esta ocasión analiza con visión crítica el origen del concepto smart city, el porqué de su éxito y rápida adopción por parte de las administraciones locales y el balance sesgado hacia el negocio y beneficio de las grandes empresas y corporaciones, y no de las necesidades de las poblaciones.

Borja afirma en su artículo que la tecnología no siempre juega a favor de los ciudadanos; y recuerda que en el año 2010 IBM lanzó una campaña publicitaria: Smart Cities Challenge; y posteriormente en 2011 lanzó un nuevo producto dirigido a un público específico y extenso, los gobiernos locales: Intelligent Center for Smarter Cities. En relación con la rápida adopción por parte de las administraciones se pregunta:

¿Cuál es el porqué de la emergencia de este mercado y del éxito del slogan Smart City? Las administraciones locales por una parte son de facto menospreciadas por los Estados. Poseen escasos recursos en relación con las demandas sociales de proximidad. Excepto las grandes ciudades el resto son minusvaloradas en el escenario público. Se les considera con frecuencia reacias a la modernización o al «localismo» lo cual no es hoy así.

En un entorno, global y local, las ciudades grandes y medias especialmente, han emergido como actores sociales, representados por los gobiernos y las elites locales. Las ciudades se hacen publicidad: globales, competitivas, sostenibles, integradoras, inteligentes, del conocimiento, participativas, atractivas, de calidad de vida, etc...

La historia nos enseña que las promesas de las grandes innovaciones tecnológicas han servido para lo bueno o para lo malo, para mayorías o para minorías, con efectos positivos o todo lo contrario. En todo caso no son neutras. Sus usos han sido casi siempre ambivalentes. Depende de quien las produce, las vende, las compra y se apropia de su uso.

Dos meses después del artículo de Borja, Joan Subirats, catedrático de ciencias políticas y también colaborador habitual de la prensa escrita, abordaba el tema bajo el título «Ciudad inteligente, ciudad compartida» (El Pais, 19 noviembre 2015). Subirats subraya en su texto el crecimiento del interés por las ciudades como espacios de innovación tecnológica y de experimentación, en momentos en que hay que repensar los formatos tradicionales de actividad económica y de la ciudad como espacio de producción y consumo. Y, en este contexto de alta densidad y de fuerte presencia simultánea de problemas y oportunidades, las, posibilidades de implementar los avances tecnológicos son innegables.

Subirats expone los argumentos que motivan el porqué del éxito del concepto de smart city. Alude a que ha sido capaz de recoger e incorporar sus potencialidades y promesas frente a los problemas, a la vez que el vocablo sugiere cambio y superación del modelo fordista. Señala, asimismo que con el nuevo concepto 
«se prometen nuevas soluciones a viejos problemas de las ciudades, pero al mismo tiempo (como otros conceptos de moda) es suficientemente ambiguo para servir de almohada a lo que cada uno pretenda. Lo que va quedando claro es que en los últimos años, el liderazgo y la inversión vienen del lado de la oferta, del lado de las grandes corporaciones que han apostado por sistemas avanzados de información y tecnologías de la comunicación y que ahora invierten en el Internet de las cosas». Entre sus ideas más significativas se seleccionan las siguientes afirmaciones:

Muchas ciudades han acogido con entusiasmo esa perspectiva, al entender que este «solucionismo tecnológico» les permitía salir o prometer salir de situaciones de bloqueo o enfrentarse de manera aparentemente innovadora a problemas enquistados.

Hoy por hoy, el modelo de smart city ha cuajado en una imagen de liderazgo tecnológico en la que predomina una lógica que calificaría de notablemente jerárquica, centralizada, tecnocrática y corporativa. Más entrada en resultados que en procesos. La perspectiva dominante apunta a una nueva gestión urbana con tres valores clave: más eficiencia, más seguridad y más sostenibilidad.

Aparentemente todos ganan, nadie pierde. Lo cierto es que no ha habido, más allá de la retórica y de experiencias limitadas, demasiado espacio para que los ciudadanos expresen lo que quieren, cómo usan o cómo pueden utilizar esta tecnología de forma autónoma y transformadora, o cómo evitar los riesgos sobre privacidad y libertad que estas innovaciones generan. $Y$ en cambio, voces más críticas apuntan a que de momento esas novedades aumentan el consumismo y la dependencia de las instituciones hacia las empresas proveedoras.

Subirats concluye afirmando que en el modelo actual de smart city predomina una lógica tecnocrática y corporativa, centrada más en resultados que en procesos. Y después de formular diversas preguntas en torno a la posibilidad de alternativas, señala que Internet permite ciudades inteligentes que partan de la inteligencia compartida por sus habitantes. Nadie mejor que ellos para innovar y mejorar. Ciudadanos inteligentes en una ciudad compartida, democrática.

La tercera y última referencia seleccionada de los medios de comunicación en torno al tema de la ciudad inteligente es la de Montserrat Pareja, profesora de economía aplicada. Se trata de un artículo en el suplemento monográfico de +Valor de El Periódico (octubre de 2015). El título del texto de Pareja es «Ciudad inteligente: del discurso al ciudadano», y en él recoge su visión del tema a partir de la premisa de que la actual revolución tecnológica es el cambio más rápido en la historia y que el impacto tecnológico transforma la economía y la movilidad, pero también el estilo de vida de las personas y la gobernanza.

Argumenta su premisa recurriendo a la perspectiva histórica, tanto de la ciudad, que se transforma y reinventa constantemente para dar cabida a las 
dinámicas de cambio económico y social, como de la evolución tecnológica. En este sentido afirma:

La revolución tecnológica supone una fuerte interconexión e interdependencia de los sistemas agentes que conforman la ciudad, determinando así una alta capacidad para transformar profundamente tanto la economía como la sociedad. En la actualidad, el mundo se enfrenta a un cambio tecnológico permanente: afecta a todos los mercados, todos los actores, todas las instituciones: es el cambio más rápido en la historia y ha venido para quedarse; no es un cambio sectorial o parcial, sino que se trata de toda una revolución transversal y universal.

La transversalidad del impacto tecnológico va más allá de la proliferación de empresas innovadoras o mejoras en la movilidad. Efectivamente se trata de transformar la economía y al movilidad, pero también el estilo de vida, las personas, el entorno y la gobernanza.

... Las ciudades compiten entre sí ¿Por qué? Porque tienen necesidad de ocupar un espacio nodal en este mundo de redes propiciar por el auge tecnológico al que estamos sometidos desde hace décadas. Compiten por atraer capital, talento, turistas.....; todo aquello que las haga merecedoras de un lugar en este espacio de flujos.

Para competir, las ciudades utilizan discursos más o menos elaborados o creíbles que venden el atractivo de la ciudad. Últimamente, son muchos los gobiernos locales que apuestan por una ciudad inteligente como leitmotiv de su estrategia competitiva. La smart city sigue vendiendo entre gobiernos locales que buscan ir más allá en su apuesta por retos de futuro, al igual que antes vendió y se apostó por la ciudad creativa, la ciudad del conocimiento o la ciudad cultural.

Pareja finaliza su aportación al monográfico sobre Smart city, afirmando que «más allá del proceso de arriba-abajo en la creación de una estrategia de ciudad, los gobiernos locales deberían facilitar canales de conectividad entre la ciudadanía y sus representantes junto con actitudes proactivas para que las sinergias creadas de abajo a arriba permitieran que la ciudad en su conjunto dé respuesta a los retos que la innovación tecnológica ha supuesto. En definitiva, el valor que tiene la tecnología en la provisión de mejor calidad de vida de los ciudadanos exige potenciar una smart city con smart citizens más allá del discurso orientado a la competitividad internacional».

\section{CONSIDERACIONES FINALES}

En el texto se han aportado unas notas sobre los procesos recientes de tecnificación de los destinos turísticos, en particular sobre los denominados destinos turísticos inteligentes, bajo el marco de la relación entre el turismo y las tecnologías de la información y la comunicación (TIC). Se han recogido datos e información reciente sobre el tema en un entorno próximo, y en concreto sobre la formación y difusión de los conceptos y discursos del paradigma smart city y 
smart destination, sobre la participación de los diversos agentes en dicho proceso y, al final, sobre la percepción por parte de los actores, con la consiguiente controversia.

Para una mejor contextualización del objetivo indicado se han incorporado referencias al turismo y a la ciudad, y en concreto al sistema turístico y al sistema funcional urbano como opción para resituar mejor el análisis y conocimiento de la relación entre turismo y tecnologías de la información y la comunicación. En este sentido se ha afirmado que el desarrollo de las redes tecnológicas ha constituido tanto un factor de modernización del espacio urbano como un factor de determinación de la jerarquía urbana y, recientemente con la irrupción de las TIC, uno de los factores clave de la función de liderazgo en un escenario de competencia entre ciudades.

A modo de consideraciones finales, además de la síntesis de los párrafos anteriores, se añaden a continuación algunas constataciones específicas. La mayoría de conceptos señalados son nuevos, surgidos en la última década o incluso en los cinco últimos años; pero las TIC y la terminología asociada a los avances tecnológicos aparecieron hace varias décadas y en su evolución se identifican varias fases diferenciadas (Macau, 2008). Por otra parte, hay que distinguir entre los nuevos conceptos «fuerza», que se benefician de la inercia de las modas y que pueden perder actualidad y vigor, de las propias tecnologías de la información y comunicación que, como señala Pareja (2015), han llegado para quedarse.

En España, el proyecto Destinos Turísticos Inteligentes que gestiona SEGITTUR, como instrumento de gestión y planificación, recuerda los planes de excelencia y dinamización de los años noventa y primeros años del nuevo siglo. Los referentes a la competitividad y sostenibilidad son coincidentes, así como la premisa de la colaboración público- privado. Incluso inicialmente el objetivo eran los destinos maduros necesitados de estrategias de revitalización. La diferencia conceptual y operativa radica en el papel que hoy desempeñan las TIC.

En la relación turismo - tecnologías de la información y comunicación y en la formación y difusión de los conceptos y del paradigma smart destination, el papel de la administración pública se observa a distintas escalas, si bien los niveles privilegiados son la administración central y la local.

Es importante distinguir, asimismo, entre los distintos ámbitos y las diversas situaciones de influencia de las TIC. En el texto se ha hecho énfasis en considerar que la realidad turística y la realidad urbana (asociada a destino) son complejas; y que plantear su relación con las TIC supone en un primer nivel situarse en un planteamiento de cierta abstracción. Para conocer el grado de afectación y uso de las TIC, el enfoque analítico ha de basarse a otra escala: la consideración de la relación de las TIC con cada uno de los componentes del 
sistema turístico o bien de los subsistemas y elementos del sistema funcional urbano. La complejidad y multiplicidad de situaciones proyecta dificultad.

En primera instancia el funcionamiento y la dinámica de la ciudad y del destino (en el marco de su relación con las TIC) pueden entenderse como el sumatorio o agregado de las diversas situaciones (afectación de la tecnología en procesos de producción-consumo, su uso por parte de residentes o visitantes, etc.). Bajo esta perspectiva, el planteamiento de una gestión pública (integral) de la ciudad o del destino se sitúa en un nivel de cierta abstracción, que correspondería a una intencionalidad que posibilitaría y limitaría, a su vez, las propias competencias (ámbitos de actuación) y capacidades de la administración local. $\mathrm{Y}$ en estas coordenadas, en la relación TIC-destino turístico cabría preguntarse qué es los circula por los mecanismos de la administración pública: lo fundamental o lo banal.

La gestión pública de la ciudad o del destino, como función administrativa y como intencionalidad, puede inspirase en diversos principios de referencia, como la «inteligencia» o la «sosteniblidad», para fundamentar el modelo de ciudad y para guiar los programas y las acciones. Asociar estos principios con los discursos sobre la gestión de la ciudad o el destino, al margen de su materialización operativa, afecta positivamente a la imagen la ciudad y a su proyección, dado el valor que hoy tienen dichos principios en el imaginario social. La adopción del paradigma de ciudad inteligente genera controversia y críticas basadas en la reclamación de la óptica social frente a su concepto tecnocrático del paradigma. La adopción del paradigma y la forma y grado de implementación aparece vinculada, finalmente, al componte político-ideológico de cada administración, de cada gobierno local.

\section{BIBLIOGRAFIA}

Blanco, J. (2015): Libro Blanco de los destinos turísticos inteligentes. Estrategias y soluciones para fomentar la innovación en el turismo digital, LID Ed., Biblioteca ALTRAN. Madrid.

BoRJA. J. (2015): «Smart cities, negocio o ciudadanía». Diario El País, 15 septiembre de 2015.

EOI (2014): Ciudades y ciudadanos en 2033. La transformación urbana de España. PwC España. www.pwc.es

Fundación Telefónica (2011): Smart Cities: un primer paso hacia el Internet de las cosas. Fundación Telefónica-Ariel. Madrid.

Generalitat Valenciana (2015): Destinos turísticos inteligentes. Manual Operativo para la Configuración de Destinos Turísticos Inteligentes. Instituto Valenciano de Tecnologías Turísticas (INVAT-TUR) e Instituto Universitario de Investigaciones Turísticas (IUTT). Valencia.

JAFARI, J. -edt- (2000): Enciclopedia del turismo. Editorial Síntesis. Madrid. 
JANÉ, C. (2015): «La ‘smart city’ baja al suelo». Diario El Periódico, 17 noviembre 2105.

Lopez de Ávila, A. y García SÁNChez, S. (2015): «Destinos turísticos inteligentes», en Economía Industrial, n. ${ }^{\circ} 395$ (2015): 61-69.

Luque Gil, A. M.a; Caro Herrero, J.L. y Zayas Fernández, B. (2014): «Los destinos turísticos inteligentes en el marco de la inteligencia territorial: conflictos y oportunidades», en Actas XIV Coloquio de Geografía, Turismo, Ocio y Recreación. Espacios turísticos e inteligencia territorial: respuestas ante la crisis (MálagaSevilla 23-25 octubre 2014), Universidad de Málaga, Universidad de Sevilla, AGE. Sevilla.

MACAU, R. (2004): «TIC: ¿para qué? (Funciones de las tecnologías de la información y la comunicación en las organizaciones)» [artículo en línea], en Revista de Universidad y Sociedad del Conocimiento (RUSC). Vol. 1, n. ${ }^{\circ} 1$. http://www.uoc. edu/rusc/dt/esp/macau0704.pdf

Marrero, R. y SAntana, M.Á., (2008): «Competitividad y calidad en los destinos turísticos de sol y playa. El caso de las Islas Canarias», en Cuadernos de Turismo, n. ${ }^{\circ}$ 22: 123-143.

Mínguez, M. a Del C. y Ruiz Jiménez, P. (2014): «Los Destinos Turísticos Inteligentes en España: ¿un proyecto institucional o el futuro del sector?», en Actas XIV Coloquio de Geografía, Turismo, Ocio y Recreación. Espacios turísticos e inteligencia territorial: respuestas ante la crisis (Málaga-Sevilla 23-25 octubre 2014), Universidad de Málaga, Universidad de Sevilla, AGE. Sevilla.

Pareja, M. (2015): «Ciudad inteligente: del discurso al ciudadano», en Monográfico +Valor del diario El Periódico, 27 octubre de 2015.

Parra, E. y Santana, A. (2014): «Tecnologías de la información y la comunicación en turismo», en A. García Sánchez (coord), 20 años de la actividad turística en España, Aecit- Ed. Síntesis. Madrid, pp. 279-292.

Pisarello, G. (2015): «Ciudad inteligente, ¿para qué?». Diario Ara, 25 noviembre en 2015.

SANCHO, A. (Dir.) (1998): Introducción al turismo. OMT. Madrid.

Subirats, J. (2015): «Ciudad inteligente, ciudad compartida». Diario El País, 19 noviembre 2015.

Vera, J.F. (Coord); López Palomeque, F.; Marchena, M. y Anton, S. (2013): Análisis territorial del turismo y planificación de destinos turísticos. Editorial Tirant Humanidades. Valencia. 